\title{
THE VALUE OF STEREOTACTIC VACUUM-ASSISTED BIOPSY IN THE INVESTIGATION OF MICROCALCIFICATIONS IN 1354 PATIENTS IN PUBLIC BRAZILIAN HOSPITAL
}

Andressa G. Amorim¹, Marcellus N. M. Ramos¹, André Mattar, Jorge Y. Shida', Luiz H. Gebrim¹

${ }^{1}$ Women's Reference Center, Hospital Pérola Byington - São Paulo (SP), Brazil.

Objectives: The gold standard for breast biopsy procedures is currently an open excision of the suspected lesion. However, an excisional biopsy inevitably makes a scar. The cost and morbidity associated with this procedure has prompted many physicians to evaluate less invasive, alternative procedures. More recently, image-guided percutaneous core-needle biopsy has become a frequently used method for diagnosing palpable and non-palpable breast lesions. Although sensitivity rates for core-needle biopsy are high, it has the disadvantage of histological underestimation, which renders the management of atypical ductal hyperplasia, papillary lesions, and fibroepithelial lesions somewhat difficult. Stereotactic vacuum assisted breast biopsy (VABB) was developed to overcome some of these negative aspects of core-needle biopsy. VABB allows for a sufficient specimen to be obtained with a single insertion and can provide a more accurate diagnosis and completely remove the lesion under real-time ultrasonic guidance. The advantage of complete lesion removal with VABB is to reduce or eliminate sampling error, to decrease the likelihood of a histological underestimation, to decrease imaging-histological discordance, to decrease the re-biopsy rate, and to diminish the likelihood of subsequent growth on follow-up, especially when stereotactic VABB is used to investigate microcalcifications. This method is expensive but cost effective when used to investigate microcalcifications classified as BI-RADS 4 and 5. Methodology: We performed a review in 1,354 patients with suspicious mammography microcalcifications, classified as BI-RADS 4 or 5 that were seen in Perola Byington Hospital from July 2012 to July 2017 in São Paulo-Brazil. We have used a Hologic Lorad Multicare Platinum Stereotactic Prone Breast Biopsy and a Surus Pearl (Hologic, Malbolrough, Massachusetts, USA), with gauge 9 probe. Four to eight fragments (median of 6) were obtained with the placement of a metal clip in the bed that the biopsy was performed, and histopathological analysis was made. Results: The histopathological study of the lesions revealed benign alterations in $956(68 \%)$ of our patients. The findings were positive for malignancy in 358 patients (29\%) and the precursor lesions were diagnosed in 40 (3\%). In 81 cases (5.9\%) the findings were discordant. The sensitivity of the method was $84.4 \%$, specificity was $96.1 \%$, false negative rate was $4.5 \%$, positive predictive value (PPV) was $89.8 \%$, negative predictive value (NPV) was 93.8\%. In literature review the sensitivity varies $91.5-100 \%$, specificity $81.9-110 \%$, false negative rate $0-3.3 \%$, PPV 92.2$100 \%$ and NPV 80.5-99.5\%. All patients with positive or discordant cases underwent surgical treatment to increase the margin or complete removal of the lesion. Conclusions: The VABB is an outpatient procedure that avoids hospital admissions for diagnostic elucidation in most of cases suspected of malignancy. It has high predictive value in both benign and malignant lesions, guiding therapeutic planning. In addition to presenting the cost lower than the surgical biopsy it indirect increases the supply of hospital beds for cancer treatment. 